\title{
No Limiar do Ensino Fundamental: Estresse, Competência e Ajustamento em Alunos do $1^{\circ}$ Ano
}

\author{
Erica Taciana dos Santos Crepaldi \\ Faculdade de Filosofia, Ciências e Letras de Ribeirão Preto, Universidade de São Paulo, \\ Ribeirão Preto, SP, Brasil \\ Marta Regina Gonçalves Correia-Zanini \\ Centro Universitário das Faculdades Associadas de Ensino - UNIFAE, São Paulo, SP, Brasil \\ Edna Maria Marturano ${ }^{1}$ \\ Faculdade de Medicina de Ribeirão Preto, Universidade de São Paulo, \\ Ribeirão Preto, SP, Brasil
}

\section{Resumo}

Este estudo objetivou comparar crianças do $1^{\circ}$ ano do Ensino Fundamental (EF), com e sem sintomas de estresse, em indicadores de ajustamento e competência relacionados ao desempenho acadêmico, social e comportamental, separadamente para meninos e meninas. Participaram da pesquisa 85 meninos e 72 meninas, bem como 25 professores, estes como informantes. As crianças responderam à Escala de Stress Infantil e ao Inventário de Estressores Escolares, e realizaram a Provinha Brasil de desempenho acadêmico. Os professores avaliaram as habilidades sociais e os problemas de comportamento externalizantes e internalizantes dos seus alunos com o Social Skills Rating System. Não houve diferença de gênero quanto ao estresse. As meninas obtiveram melhores resultados em desempenho acadêmico e habilidades sociais; os meninos apresentaram mais problemas externalizantes. Na comparação entre crianças com estresse e sem estresse, do mesmo sexo, meninos com estresse estão em desvantagem em todas as variáveis avaliadas. Entre as meninas se verifica tendência semelhante, porém com menos variáveis envolvidas (seis das 10 variáveis). Os resultados sugerem que crianças mais competentes apresentam maior facilidade para se ajustarem às demandas adaptativas da transição. Elas percebem as tarefas adaptativas como menos estressantes, o que supostamente as torna menos expostas aos efeitos adversos do estresse.

Palavras-chave: Estresse infantil, transição escolar, ensino fundamental, habilidades sociais.

\section{Elementary School Entry: Stress, Competence and Adjustment in First Grade Students}

\footnotetext{
Abstract

This study aimed to compare children from the $1^{\text {st }}$ year of Elementary Education (EE) with or without stress symptoms regarding indicators of adjustment and competence related to academic, social, and

Endereço para correspondência: Universidade de São Paulo, Faculdade de Medicina de Ribeirão Preto, Departamento de Neurociências e Ciências do Comportamento, Bloco da Saúde Mental, Rua Tenente Catão Roxo, 2650, Ribeirão Preto, SP, Brasil, 14051-140. E-mail:emmartur@fmrp.usp.br

As autoras agradecem às crianças e aos professores participantes, às famílias que autorizaram a participação de suas crianças e às escolas que aceitaram e viabilizaram a realização desta pesquisa. Também agradecem a contribuição das psicólogas Jéssica Castro e Daniele Teixeira na coleta de dados e o apoio do Conselho Nacional de Desenvolvimento Científico e Tecnológico (CNPq) e da Coordenação de Aperfeiçoamento de Pessoal de Nível Superior (CAPES) e Fundação de Amparo à Pesquisa do Estado de São Paulo (FAPESP).
} 
behavioral domains, separately for boys and girls. The participants were 85 boys and 72 girls. Twenty-five teachers participated as informants. The children answered the Childhood Stress Scale, the School Hassles Inventory and the academic test Provinha Brasil. The teachers evaluated the social skills and externalizing, and internalizing behavior problems of the children using the Social Skills Rating System. There was no gender difference in self-reported stress symptoms. Girls outperformed boys in the academic achievement test. The teachers rated the girls as more socially skilled and the boys with more externalizing behavior problems. In the comparison of the children with or without stress symptoms, boys with stress were disadvantaged in all variables. Among girls, a similar pattern was found for six out of the 10 variables studied. The results suggest that children that are more competent deal more easily with the transitional requirements of the first year in EE. These children find the adaptive tasks less stressful, which appears to make them less susceptible to the adverse effects of stress.

Keywords: Childhood stress, transition school, elementary school, social skills.

\section{En el Umbral dela Escuela Primaria: Estrés, Competencia y el Ajuste de los Estudiantes}

\section{Resumen}

Este estudio comparó los niños de primero año de la Escuela Primaria (EP) con y sin síntomas de estrés en los indicadores de ajuste y de habilidades relacionadas con el rendimiento académico, social y de comportamiento, de forma separada para niños y niñas. Los participantes fueron 157 estudiantes (72 niñas) y sus profesoras $(N=25)$. Los niños respondieron a la Escala de Estrés Infantil, al Inventario Estresores Escolar y llevaron a cabo una prueba de rendimiento académico (Provinha Brasil). Las profesoras evaluaran habilidades sociales, externalización y internalización de sus estudiantes (Social Skills Rating System). No hubo diferencias entre niñas y niños en relación al estrés. Las niñas tuvieron promedios más altos en el rendimiento académico y en las habilidades sociales. Los niños tuvieron más problemas de externalización. Entre los estudiantes del mismo sexo con y sin síntomas de estrés, los niños con estrés están en desventaja en todas las variables. Las niñas presentan tendencias similares, pero con menos variables involucradas (seis de las 10 variables). Los resultados sugieren que los niños más competentes logran mejor adaptarse a las demandas de la transición y perciben las tareas de adaptación como menos estresantes, lo que supuestamente los hace menos expuestos a los efectos adversos del estrés.

Palabras clave: Estrés infantil, transición de la escuela, Escuela Primaria, habilidades sociales.

A noção tradicional da infância como um período livre de demandas externas, responsabilidades e estresse tem sido confrontada nas últimas décadas pela pesquisa sobre estresse infantil (Byrne, Thomas, Burchell, Olive, \& Mirabito, 2011). De acordo com Lipp (2000) no curso de seu desenvolvimento intelectual, emocional e afetivo, a criança se depara com situações desafiadoras que podem vir a exceder a sua capacidade, ainda imatura, para lidar com elas, tornando-a vulnerável ao estresse.

Segundo Lipp e Lucarelli (2008), o estresse infantil se assemelha ao dos adultos e envolve uma reação do organismo com componentes fí- sicos (dores de cabeça, dores abdominais, alterações do apetite, diarreia, gagueira, etc.) e/ou psicológicos (desânimo, ansiedade, birra, choro excessivo, terror noturno, etc.) frente a qualquer estímulo que irrite, amedronte e/ou faça feliz a pessoa, isto é, qualquer fato novo que exija adaptação. As autoras propõem que se avalie o estresse de acordo com o conjunto de sintomas que apresenta e a fase de evolução destes: fases de Alerta ou Alarme, Defesa ou Resistência, Quase-exaustão e Exaustão, considerada a fase mais aguda do estresse.

Estudos brasileiros sobre avaliação e incidência de estresse infantil são escassos; no entan- 
to, três pesquisas realizadas no contexto escolar indicam certa prevalência de estresse, merecedora de atenção. No estudo de Vilela (1995) foram avaliados 40 alunos de $1^{\text {a }}$ a $4^{\text {a }}$ série do ensino fundamental $(\mathrm{EF})$ de escolas públicas ou particulares, e $62 \%$ deles apresentaram sintomas de estresse, na maioria em fase de alerta. Tanganelli e Lipp (1998), avaliando 158 alunos de $1^{\text {a }}$ a $4^{\mathrm{a}}$ série, identificaram sintomas compatíveis com a presença de estresse em $55 \%$ da amostra. E, no estudo de Enumo, Ferrão e Ribeiro (2006), apresentaram indicativos de estresse $21 \%$ dos 60 alunos avaliados, com idades entre 8 e 14 anos.

As investigações sobre o estresse, tanto de adultos quanto de crianças, têm apontado que as tensões cotidianas detêm maior poder de avaliação do estresse na predição de sintomas e disfunções psicológicas que os eventos de vida de característica imprevisível, incontrolável, excepcional e traumática (Bridley \& Jordan, 2012). As tensões cotidianas, conhecidas na literatura internacional como "daily hassles", compreendem as demandas irritantes, frustrantes e angustiantes que em algum grau caracterizam as transações diárias com o ambiente, podendo afetar o bem-estar físico e emocional de adultos, adolescentes e crianças (Bridley \& Jordan, 2012; Escobar et al., 2013; Kanner, Coyne, Schaefer, \& Lazarus, 1981).

Dentre as principais fontes de tensões cotidianas na infância encontra-se a escola, lugar onde as crianças passam grande parte do seu tempo. A escola é vista por muitos alunos como uma fonte importante de tensões diárias (Eriksson \& Sellströn, 2010; Rende \& Plomin,1992). As tensões do ambiente escolar estão presentes nas demandas acadêmicas, nos relacionamentos com os colegas e professores, nas notas baixas e na realização de muitas atividades depois da escola (Escobar et al., 2013; Vanaelst, De Vriendt, Huybrechts, Rinaldi, \& De Henauw, 2012).

Tensões cotidianas na escola têm sido associadas a sintomas de estresse em diferentes culturas. Na Suécia, Hjern, Alfven e Östberg (2008) encontraram associação forte entre a percepção de estressores escolares e queixas psicossomáticas em crianças e adolescentes de 10 a 18 anos, ao passo que Eriksson e Sellströn (2010), com alunos de 11 a 15 anos, identificaram a percepção de demandas acadêmicas como um preditor de queixas subjetivas de saúde. No estudo de Hesketh et al. (2010), realizado com 1291 crianças chinesas com idade entre nove e 12 anos, os participantes identificaram múltiplos estressores na escola e todos os estressores estavam significativamente associados a sintomas psicossomáticos. Em pesquisa realizada com crianças brasileiras que frequentavam a antiga $1^{\text {a }}$ série do EF, Trivellato-Ferreira e Marturano (2008) encontraram correlação positiva entre sintomas de estresse e percepção de tensões cotidianas na escola.

Além das tensões comumente presentes no ambiente escolar, parece haver um aumento da vulnerabilidade ao estresse em momentos de transição escolar (Marturano, 2008). Especificamente, o ingresso no EF configura a transição entre os estágios pré-escolar e escolar, momento em que a criança se depara com as tarefas de desenvolvimento do novo estágio: ter sucesso no aprendizado acadêmico, formar laços de amizade com os pares e aderir às normas da sociedade para conduta moral e comportamento pró-social, entre outras (Masten \& Coatsworth, 1998).

Acresce, porém, que os desafios do ingresso no EF não se limitam à exigência das tarefas de desenvolvimento da idade escolar. A mudança de nível escolar carreia tarefas adaptativas importantes, dentre as quais se sobressaem as de lidar com um novo ambiente, relacionar-se com adultos ainda desconhecidos, conquistar aceitação em um novo grupo de pares e atender às exigências do papel de estudante, traduzidas nas expectativas dos adultos e formalizadas por meio de avaliações periódicas de progresso. Estas tarefas tornam-se ainda mais desafiadoras quando a criança não frequentou a educação infantil (EI), ou quando, mesmo a tendo frequentado, precisa mudar de escola para ingressar no EF (Marturano, 2008; Trivellato-Ferreira \& Marturano, 2008). Impõe-se, então, como tema relevante de pesquisa, a questão dos possíveis efeitos dessa conjunção de desafios sobre o funcionamento adaptativo da criança que ingressa no EF.

O funcionamento adaptativo na infância pode ser avaliado em termos de competência e 
disfunção. A competência refere-se ao grau em que a criança é capaz de resolver de forma eficiente as tarefas de desenvolvimento e os desafios presentes no seu ambiente, resultando numa boa adaptação e não necessariamente num desempenho excelente. Já a disfunção, implicando em problemas no funcionamento adaptativo, compreende a dificuldade recorrente em manter comportamentos de autocontrole (Gardinal-Pizato, Marturano, \& Fontaine, 2014; Masten \& Coatsworth, 1998).

As pesquisas sobre competência infantil nos anos do EF têm investigado os domínios acadêmico e social devido à relevância desses para as tarefas de desenvolvimento da idade escolar (Chen, Huang, Chang, Wang, \& Li, 2010; Correia-Zanini, 2013). A competência no domínio acadêmico é comumente estimada por provas objetivas de habilidades escolares e avaliação do professor (Correia-Zanini, 2013). No que se refere ao domínio social, a competência é frequentemente operacionalizada por meio do constructo habilidades sociais, que abarca um conjunto de classes de comportamentos apresentados pelo indivíduo em situações sociais que propiciam um relacionamento interpessoal saudável e produtivo e implicam em algum controle das próprias emoções (Gardinal-Pizato et al., 2014). São exemplos de classes de habilidades sociais a assertividade, solução de problemas interpessoais, cooperação, empatia, expressividade emocional e habilidades sociais acadêmicas (Del Prette \& Del Prette, 2005; Gardinal-Pizato et al., 2014).

No que se refere aos indicadores de disfunções, estes têm sido operacionalizados por meio do termo ajustamento comportamental que, por sua vez, está associado a dois fatores amplos: comportamentos externalizantes e internalizantes. Os comportamentos externalizantes incluem problemas com atenção e autorregulação, e comportamentos antissociais e agressivos, enquanto os comportamentos internalizantes incluem depressão, ansiedade, isolamento, sentimentos de inferioridade e queixa somáticas (Achenbach \& Edelbrock, 1979).

$\mathrm{Na}$ tentativa de identificar condições associadas ao impacto das demandas iniciais do EF sobre o funcionamento adaptativo dos alunos, pode-se supor que crianças com indicadores mais favoráveis de funcionamento adaptativo estariam mais protegidas frente essas demandas, ao passo que crianças com funcionamento mais pobre estariam mais vulneráveis ao estresse da transição. Essas relações são sugeridas pelos resultados do estudo de Trivellato-Ferreira e Marturano (2008), que encontraram, em crianças que iniciavam o EF, correlação negativa entre sintomas de estresse e medidas de competência acadêmica e ajustamento. Além disso, a partir do exposto e da literatura sobre diferenças de sexo, os meninos estariam mais vulneráveis ao estresse nos anos iniciais do EF, dado que nessa fase de escolaridade eles apresentam desempenho acadêmico mais pobre (Grimm, Steele, Mashburn, Burchinal, \& Pianta, 2010; Matthews, Kizzie, Rowley, \& Cortina, 2010), habilidades sociais menos desenvolvidas (Bandeira, Rocha, Freitas, Del Prette, \& Del Prette, 2006; Matthews et al., 2010) e mais problemas de comportamento externalizantes (Fanti \& Henrich, 2010; Robbers et al., 2010; Silver, Measelle, Armstrong, \& Essex, 2010). Porém, há também alguma evidência empírica de que as meninas em geral relatam mais sintomas de estresse (Lipp, Arantes, Buriti, \& Witzig, 2002), embora essa evidência não seja consensual (Pacanaro \& Di Nucci, 2005).

Nesse contexto se insere o presente estudo, que teve como objetivo comparar crianças avaliadas com sintomas de estresse e sem sintomas de estresse em relação ao desempenho acadêmico, às habilidades sociais, aos problemas de comportamento e à percepção de estressores escolares, no $1^{\circ}$ ano do EF. São consideradas crianças com sintomas de estresse aquelas que se encontram em alguma das quatro fases evolutivas do estresse conforme o modelo sugerido por Lipp e Lucarelli (2008) na sua Escala de Stress Infantil - ESI, e crianças sem estresse aquelas que não se enquadram em nenhuma destas fases. Vale ressaltar, portanto, que não se trata de diagnóstico de sintomas de estresse em nível clínico, mas sim da presença de estresse em alguma fase.

A hipótese de trabalho foi a de que crianças com estresse apresentariam menor desempenho acadêmico, menor repertório de habilidades sociais, mais problemas de comportamento e maior 
percepção de estressores escolares em relação às crianças sem estresse. Dadas as diferenças de sexo apontadas na literatura, foram preliminarmente investigadas associações com essa variável; além disso, a hipótese de trabalho foi testada separadamente para meninos e meninas.

\section{Método}

\section{Considerações Éticas}

Esta pesquisa é parte de projeto aprovado pelo Comitê de Ética em Pesquisa da Faculdade de Filosofia, Ciências e Letras de Ribeirão Preto da Universidade de São Paulo. Atende às normas dispostas na Resolução n ${ }^{\circ}$ 196/96 da Comissão Nacional de Ética em Pesquisa (CONEP) e ao disposto na Resolução no 016/2000 do Conselho Federal de Psicologia. Os participantes adultos assinaram o Termo de Consentimento Livre e Esclarecido - TCLE; no caso de participante criança, um dos responsáveis assinou o TCLE e o participante deu seu prévio consentimento verbal.

\section{Participantes}

A amostra original deste estudo foi constituída por sorteio em 25 turmas do $1^{\circ}$ ano do $\mathrm{EF}$, em escolas municipais de uma cidade do interior de São Paulo com aproximadamente 111 mil habitantes (Correia-Zanini, 2013). Em cada turma foram sorteados 13 alunos, resultando em uma amostra presumida de 325 participantes. Todos os responsáveis foram consultados e 181 deram seu consentimento para a participação da criança. Do presente estudo participaram 157 alunos dessa amostra, que atenderam ao critério de dois anos de experiência prévia na EI, sendo 85 meninos e 72 meninas, com idade entre cinco anos e oito meses e sete anos e seis meses (média 6 anos e 10 meses, $D P 4$ meses). Também participaram do estudo, como informantes, seus respectivos professores do $1^{\circ}$ ano do EF, num total de 25.

\section{Local}

Os dados foram coletados em sete dentre as 15 escolas municipais que oferecem EF. A se- leção das escolas foi realizada juntamente com técnicos da secretaria municipal de educação, visando representar diferentes regiões da cidade, sendo uma escola na região central, duas próximas ao centro e quatro em bairros mais afastados, totalizando 25 turmas.

\section{Instrumentos}

Escala de Stress Infantil - ESI. A ESI tem como objetivo identificar a frequência com que crianças de seis a 14 anos experimentam sintomas de estresse e quais tipos de reação caracterizam tais sintomas (Lipp \& Lucarelli, 2008). A escala é composta por 35 itens, cada qual acompanhado de uma escala analógica do tipo Likert, em forma de círculo dividido em quadrantes. A pontuação corresponde ao número de quadrantes do círculo que a criança pintou. Não pintar o círculo corresponde a - nunca acontece - 0 ponto; pintar uma parte - acontece um pouco - 1 ponto; pintar duas partes - acontece às vezes, 2 pontos; pintar três partes -acontece quase sempre -3 pontos; pintar 4 partes - acontece sempre - 4 pontos. A soma de pontos de todos os itens resulta no escore total de sintomas apresentados pela criança. Com este escore também é possível identificar a fase do estresse: de 0 a 39,5 - sem estresse; de 39,6 a 59,4 - fase de alerta; de 59,5 a 79,4 pontos - fase de resistência; 79,5 a 99,3 ou sete círculos com todas as partes pintadas fase de quase exaustão; acima de 99,3 - fase de exaustão. No presente estudo, utilizou-se uma classificação dicotômica entre as pontuações 39,5 e 39,6 para discriminar crianças sem sintomas de estresse e crianças com sintomas nas fases de alerta, resistência, quase exaustão ou exaustão.

Provinha Brasil 2009. A avaliação denominada Provinha Brasil foi elaborada pelo Instituto Nacional de Estudos e Pesquisas Educacionais Anísio Teixeira (INEP, 2009) e tem como objetivo diagnosticar o nível de alfabetização das crianças matriculadas no $2^{\circ}$ ano do EF das escolas públicas brasileiras. Ela é composta por um exemplo de questão que possibilita ensinar aos alunos como deverão responder ao teste e por outras 24 questões de múltipla escolha formuladas para avaliar o desempenho. As questões 
avaliativas são sequenciadas em níveis crescentes de exigência de habilidades, desde as mais básicas até as mais avançadas. As questões são de três tipos: Tipo 1 - Questões cujos comandos e alternativas precisam ser totalmente lidos pelo aplicador; Tipo 2 - Questões nas quais o aplicador lê apenas os comandos; Tipo 3 - Questões em que o aplicador lê apenas a orientação inicial para avisar aos alunos que terão que ler sozinhos o texto, os enunciados e as alternativas. Cada questão acertada recebe um ponto e a pontuação total é referente à soma dos itens que a criança acertou, variando, portanto, entre 0 e 24 (http:// provinhabrasil.inep.gov.br).

Sistema de Avaliação de Habilidades Sociais. Utilizou-se a adaptação brasileira do $S o$ cial Skills Rating System - SSRS-BR - versão para Professores (Bandeira, Del Prette, Del Prette, \& Magalhães, 2009). O questionário destinado aos professores compreende três escalas: habilidades sociais, com cinco fatores (cooperação com pares, asserção positiva, responsabilidade/ cooperação, autodefesa e autocontrole); problemas de comportamento, em dois fatores (internalizantes e externalizantes); competência acadêmica, composta por nove itens, não utilizada neste estudo. Para avaliar a frequência de habilidades sociais e de comportamentos problemáticos há três alternativas de resposta: "nunca" $(0)$, "algumas vezes" (1) e "muito frequente" (2). O escore total de cada escala ou fator é o resultado da soma obtida nos itens correspondentes. Bandeira et al. (2009) relatam índices satisfatórios de consistência interna e estabilidade temporal para a adaptação brasileira das escalas. A estrutura fatorial utilizada neste estudo foi a obtida por meio de Análise Fatorial Confirmatória realizada por Correia-Zanini (2013).

Inventário de Estressores Escolares - IEE. O IEE visa investigar situações perturbadoras ou irritantes relacionadas à vida escolar, em quatro domínios: desempenho escolar, relação família-escola, relação com os pares, outras demandas da vida escolar (Marturano \& Gardinal, 2008; Trivellato-Ferreira \& Marturano, 2008). Apresentado individualmente à criança, é composto por trinta itens. Para cada situação apresentada, a criança responde se aquilo aconteceu com ela durante o ano letivo; caso tenha acontecido, informa ainda o quanto a situação a aborreceu. Para a medida de exposição aos estressores, atribui-se valor zero ao item que, segundo a criança, não ocorreu, e um para a situação que ocorreu. Para a medida de impacto, cada item ocorrido recebe o acréscimo de zero, um, dois ou três pontos, de acordo com a intensidade do efeito relatado pela criança em uma escala de quatro pontos: nada, só um pouco, mais ou menos, muito. No presente estudo, o IEE foi utilizado com a estrutura fatorial de duas dimensões, identificada por Gardinal-Pizato (2010) e revisada por Correia-Zanini (2013): (a) estressores associados ao papel de estudante, com 10 itens referentes ao desempenho escolar, à relação família-escola e ao professor; (b) estressores derivados das relações interpessoais na escola, com 7 itens do relacionamento com os pares e com adultos.

\section{Procedimento}

A coleta de dados com as crianças e as professoras se deu nos meses de novembro e dezembro de 2010, no horário de aula e em espaços cedidos pelas escolas. As crianças responderam individualmente aos instrumentos IEE e ESI. A Provinha Brasil foi aplicada coletivamente pela segunda autora com a ajuda de uma bolsista de apoio técnico, em turmas de 5 a 20 alunos, conforme espaço disponível para a atividade. As professoras foram orientadas sobre o preenchimento dos formulários do SSRS.

Para formação dos grupos com e sem estresse foi levantada a distribuição percentual de alunos em cada fase de estresse, a partir da Escala de Stress Infantil. A distribuição percentual pelas fases do estresse foi a seguinte: sem estresse, $42,7 \%(n=67)$; fase de alerta, $11,5 \%$; fase de resistência, $1,9 \%$; fase de quase exaustão, $42,7 \%$; fase de exaustão, $1,3 \%$. De acordo com a presença de sintomas de estresse, foram constituídos dois grupos, resultando em 90 crianças no grupo com estresse (57\%) e 67 no grupo sem estresse. Os grupos foram comparados em termos de distribuição e sexo por meio do teste Qui-quadrado. Uma vez confirmados os pressupostos para utilização de estatística paramétrica, foi aplicado o teste $t$ de Student, adotando-se o nível de signifi- 
cância de $p \leq 0,05$, para compará-los em relação ao desempenho acadêmico, habilidades sociais, problemas de comportamento e percepção de estressores escolares. Para aferição da magnitude de efeito entre as comparações, foi utilizado o cálculo do $d$ de Cohen, com as seguintes faixas de magnitude, de acordo com Marôco (2011, p. 251): pequena, quando $d \leq 0,2 ;$ média, quando $d$ varia entre 0,2 e 0,5 ; elevada, quando $d$ se situa entre 0,5 e 1,0 ; muito elevada, se $d>1$.

\section{Resultados}

Não se encontrou diferença significativa entre meninas e meninos em relação à presença de estresse (meninas, $51 \%$; meninos, $62 \% ; \chi^{2}(1)=$ $0,422, p>0,05)$. Na Tabela 1 se encontram os resultados das demais comparações entre sexos. A tabela apresenta as médias e desvios padrão para meninos e meninas, bem como os valores de $t \mathrm{e}$ das probabilidades associadas.

\section{Tabela 1}

Comparação entre Meninos e Meninas nas Variáveis Avaliadas no $1^{\circ}$ ano

\begin{tabular}{|c|c|c|c|}
\hline \multirow{2}{*}{ Variáveis } & $\begin{array}{c}\text { Meninos } \\
n=85\end{array}$ & $\begin{array}{c}\text { Meninas } \\
n=72\end{array}$ & \multirow{2}{*}{$t$} \\
\hline & $\begin{array}{l}\text { Média } \\
(d p)\end{array}$ & $\begin{array}{l}\text { Média } \\
(d p)\end{array}$ & \\
\hline \multirow{2}{*}{ Desempenho Acadêmico } & 12,44 & 14,83 & \multirow{2}{*}{$-3,229 *$} \\
\hline & $(4,68)$ & $(4,59)$ & \\
\hline \multirow{2}{*}{ Asserção Positiva } & & & \multirow{2}{*}{$-2,940^{*}$} \\
\hline & $(2,86)$ & $(2,75)$ & \\
\hline \multirow{2}{*}{ Autocontrole } & 9,46 & 10,86 & \multirow{2}{*}{$-2,595^{*}$} \\
\hline & $(3,58)$ & $(3,11)$ & \\
\hline \multirow{2}{*}{ Autodefesa } & 2,96 & 3,44 & \multirow{2}{*}{$-2,003^{*}$} \\
\hline & $(1,49)$ & $(1,50)$ & \\
\hline \multirow{2}{*}{ Responsabilidade e Cooperação } & 9,19 & 11,06 & \multirow{2}{*}{$-3,573 * *$} \\
\hline & $(3,82)$ & $(2,70)$ & \\
\hline \multirow{2}{*}{ Cooperação com pares } & 3,12 & 4,07 & \multirow{2}{*}{$-3,730^{*}$} \\
\hline & $(1,66)$ & $(1,51)$ & \\
\hline \multirow{2}{*}{ PC - Externalizante } & 5,82 & 4,06 & \multirow{2}{*}{$3,013^{*}$} \\
\hline & $(3,83)$ & $(3,45)$ & \\
\hline \multirow[t]{2}{*}{ PC - Internalizante } & 2,92 & 2,85 & \multirow{2}{*}{0,192} \\
\hline & $(2,22)$ & $(2,37)$ & \\
\hline \multirow{2}{*}{$\begin{array}{l}\text { Percepção de estressores escolares - F1: Tensões } \\
\text { relacionadas ao papel de estudante }\end{array}$} & 13,38 & 11,82 & \multirow{2}{*}{1,238} \\
\hline & $(7,98)$ & $(7,69)$ & \\
\hline \multirow{2}{*}{$\begin{array}{l}\text { Percepção de estressores escolares - F2: Tensões } \\
\text { cotidianas nas relações interpessoais }\end{array}$} & 8,19 & 10,01 & \multirow{2}{*}{$-1,651$} \\
\hline & $(6,73)$ & $(7,09)$ & \\
\hline \multirow[t]{2}{*}{ Sintomas de estresse } & 49,06 & 46,03 & \multirow[t]{2}{*}{0,818} \\
\hline & $(24,61)$ & $(21,26)$ & \\
\hline
\end{tabular}

${ }^{*} p<0,05 ; * * p<0,001$. 
Os resultados na Tabela 1 indicam diferenças de sexo nas variáveis relacionadas ao desempenho acadêmico e às habilidades sociais. $\mathrm{O}$ grupo das meninas apresentou maiores médias no desempenho acadêmico, com tamanho de efeito médio $(d=-0,52)$, e em todas as habilidades sociais avaliadas, sendo que em responsabilidade e cooperação com pares apresentaram um tamanho de efeito elevado $(-0,56$ e 0,60 , respectivamente), e para as demais habilidades a medida foi média, com $d=-0,32$ para autodefesa, $d=$ 0,42 para autocontrole e $d=-0,47$ para asserção positiva. Também a medida de comportamento externalizante diferenciou os grupos, com maior média para o dos meninos $(d=0,48)$. Não se observaram diferenças nas medidas de internalização e percepção de estressores.

Os resultados das comparações entre crianças com e sem estresse são mostrados nas Tabelas 2 e 3 , respectivamente para meninos e meninas.

Tabela 2

Comparação entre Meninos Com Estresse e Sem Estresse nas Outras Variáveis Avaliadas no $1^{\circ}$ ano

\begin{tabular}{|c|c|c|c|}
\hline \multirow{2}{*}{ Variáveis } & $\begin{array}{l}\text { Com estresse } \\
\qquad \begin{array}{l}n=53\end{array}\end{array}$ & $\begin{array}{c}\text { Sem estresse } \\
\quad n=32\end{array}$ & \multirow[t]{2}{*}{$t$} \\
\hline & $\begin{array}{l}\text { Média } \\
(d p)\end{array}$ & $\begin{array}{l}\text { Média } \\
(d p)\end{array}$ & \\
\hline \multirow{2}{*}{ Desempenho Acadêmico } & 11,23 & 14,44 & $-3,236^{*}$ \\
\hline & $(4,25)$ & $(4,72)$ & \\
\hline \multirow{2}{*}{ Asserção Positiva } & 7,57 & 9,19 & $-2,617^{*}$ \\
\hline & $(2,78)$ & $(2,74)$ & \\
\hline \multirow{2}{*}{ Autocontrole } & 8,68 & 10,75 & $-2,676^{*}$ \\
\hline & $(3,89)$ & $(2,58)$ & \\
\hline \multirow{2}{*}{ Autodefesa } & 2,70 & 3,41 & $-2,167^{*}$ \\
\hline & $(1,46)$ & $(1,46)$ & \\
\hline \multirow{2}{*}{ Responsabilidade e Cooperação } & 8,06 & 11,06 & $-3,781^{* *}$ \\
\hline & $(3,81)$ & $(3,07)$ & \\
\hline \multirow{2}{*}{ Cooperação com pares } & 2,74 & 3,75 & $-2,846^{*}$ \\
\hline & $(1,58)$ & $(1,60)$ & \\
\hline \multirow{2}{*}{ PC - Externalizante } & 6,94 & 3,97 & $3,722 * *$ \\
\hline & $(3,88)$ & $(2,98)$ & \\
\hline \multirow{2}{*}{ PC - Internalizante } & 3,30 & 2,28 & $2,093 *$ \\
\hline & $(2,32)$ & $(1,92)$ & \\
\hline \multirow{2}{*}{$\begin{array}{l}\text { Percepção de estressores escolares - F1: Tensões } \\
\text { relacionadas ao papel de estudante }\end{array}$} & 15,36 & 10,09 & $3,093 *$ \\
\hline & $(8,05)$ & $(6,78)$ & \\
\hline \multirow{2}{*}{$\begin{array}{l}\text { Percepção de estressores escolares - F2: Tensões } \\
\text { cotidianas nas relações interpessoais }\end{array}$} & 10,04 & 5,13 & $3,467 * *$ \\
\hline & $(6,51)$ & $(6,02)$ & \\
\hline
\end{tabular}

${ }^{*} p<0,05 ; * * p<0,001$. 
Tabela 3

Comparação entre Meninas Com Estresse e Sem Estresse nas Outras Variáveis Avaliadas no $1^{\circ}$ Ano

\begin{tabular}{|c|c|c|c|}
\hline \multirow{2}{*}{ Variáveis } & $\begin{array}{c}\text { Com estresse } \\
\qquad n=37\end{array}$ & $\begin{array}{l}\text { Sem estresse } \\
\qquad n=35\end{array}$ & \multirow[t]{2}{*}{$t$} \\
\hline & $\begin{array}{l}\text { Média } \\
(d p)\end{array}$ & $\begin{array}{l}\text { Média } \\
(d p)\end{array}$ & \\
\hline \multirow{2}{*}{ Desempenho Acadêmico } & 13,43 & 16,31 & $-2,787^{*}$ \\
\hline & $(3,86)$ & $(4,88)$ & \\
\hline \multirow{2}{*}{ Asserção Positiva } & 9,27 & 9,74 & $-0,727$ \\
\hline & $(2,68)$ & $(2,83)$ & \\
\hline \multirow{2}{*}{ Autocontrole } & 9,76 & 12,03 & $-3,342 * *$ \\
\hline & $(3,39)$ & $(2,31)$ & \\
\hline \multirow{2}{*}{ Autodefesa } & 3,38 & 3,51 & $-0,382$ \\
\hline & $(1,52)$ & $(1,50)$ & \\
\hline \multirow{2}{*}{ Responsabilidade e Cooperação } & 10,30 & 11,86 & $-2,558^{*}$ \\
\hline & $(2,87)$ & $(2,29)$ & \\
\hline \multirow{2}{*}{ Cooperação com pares } & 4,08 & 4,06 & $-0,067$ \\
\hline & $(1,46)$ & $(1,59)$ & \\
\hline \multirow{2}{*}{ PC - Externalizante } & 5,00 & 3,06 & $2,472 *$ \\
\hline & $(3,86)$ & $(2,67)$ & \\
\hline \multirow{2}{*}{ PC - Internalizante } & 3,05 & 2,63 & 0,761 \\
\hline & $(2,37)$ & $(2,38)$ & \\
\hline \multirow{2}{*}{$\begin{array}{l}\text { Percepção de estressores escolares - F1: Tensões } \\
\text { relacionadas ao papel de estudante }\end{array}$} & 14,59 & 8,89 & $3,368^{* *}$ \\
\hline & $(7,79)$ & $(6,49)$ & \\
\hline \multirow{2}{*}{$\begin{array}{l}\text { Percepção de estressores escolares - F2: Tensões } \\
\text { cotidianas nas relações interpessoais }\end{array}$} & 11,95 & 7,97 & $2,458^{* *}$ \\
\hline & $(7,59)$ & $(5,98)$ & \\
\hline
\end{tabular}

$* p<0,05 ; * * p<0,001$.

Como se observa na Tabela 2, meninos com estresse estão em desvantagem em relação aos pares sem estresse, em todas as variáveis avaliadas. Eles têm médias menores, com tamanho de efeito elevado, no indicador de desempenho acadêmico $(d=-0,71)$, nas habilidades sociais de asserção positiva $(d=-0,59)$, autocontrole $(d=-$ $0,63)$, responsabilidade e cooperação $(d=-0,87)$ e cooperação com pares $(d=-0,64)$. Para a habilidade social de autodefesa, eles também apresentaram maior média, porém, com tamanho de efeito médio $(d=-0,49)$. Nas medidas de com- portamento externalizante $(d=0,86)$, de tensões relacionadas ao papel de estudante $(d=0,71)$ e de tensões cotidianas nas relações interpessoais $(d=0,78)$, eles apresentaram maiores médias com diferenças significativas e tamanho de efeito elevado. Em comportamentos internalizantes a diferença teve tamanho de efeito médio $(d=0,48)$.

Entre as meninas se verifica tendência semelhante, porém com menos variáveis envolvidas, todas com tamanho de efeito elevado. Os resultados na Tabela 3 mostram a presença de estresse associada a um pior desempenho aca- 
dêmico $(d=-0,65)$, a menos habilidades sociais de autocontrole $(d=-0,78)$ e responsabilidade/ cooperação $(d=-0,60)$, a mais problemas de comportamento externalizantes $(d=-0,58)$ e a uma percepção de maior exposição a estressores escolares em tensões relacionadas ao papel de estudante $(d=-0,80)$ e às relações interpessoais $(d=-0,58)$. Os grupos não diferiram quanto a alguns indicadores avaliados pelo professor no SSRS: habilidades sociais (asserção positiva, autodefesa, cooperação com pares) e problemas de comportamento internalizantes.

\section{Discussão}

O presente estudo focalizou o estresse da criança no $1^{\circ}$ ano do EF. Seu objetivo foi comparar crianças avaliadas com sintomas de estresse e sem sintomas de estresse em relação ao desempenho acadêmico, às habilidades sociais, aos problemas de comportamento e à percepção de estressores escolares, no $1^{\circ}$ ano do EF, separadamente para meninos e meninas. A hipótese foi de que crianças com estresse apresentariam menor desempenho acadêmico, menos habilidades sociais, mais problemas de comportamento e maior percepção de estressores escolares em comparação às crianças sem estresse.

Já na composição dos grupos, verificou-se que $57 \%$ dos alunos do $1^{\circ}$ ano apresentavam sintomas de estresse em algum nível, sendo $43 \%$ na fase de quase exaustão, uma das fases mais graves do estresse (Lipp \& Lucarelli, 2008). Este índice é preocupante por se tratar de uma amostra composta somente por crianças que frequentaram a EI, uma condição que atenua os efeitos do estresse associado à transição. A literatura aponta a passagem pela EI associada a maiores níveis de desempenho e ajustamento da criança nos resultados do primeiro ano do EF e uma maior aceitação pelos colegas, avaliações estas que correspondem a uma vivência subjetiva de menor estresse relacionado à escola (Marturano \& Gardinal, 2008; Pereira, Marturano, GardinalPizato, \& Fontaine, 2011; Trivellato-Ferreira \& Marturano, 2008).

Não houve diferença significativa entre meninos e meninas em relação à presença de sintomas de estresse. Este resultado vai ao encontro dos obtidos por outros autores que também utilizaram a Escala de Stress Infantil para avaliar sintomas de estresse em escolares, seja na $1^{\text {a }}$ (Trivellato-Ferreira \& Marturano, 2008) ou na $4^{\mathrm{a}}$ série do antigo EF de oito anos (Pacanaro $\&$ Di Nucci, 2005). Em contrapartida, difere daqueles obtidos por Lipp et al. (2002) em amostra de escolares com idades entre 7 e 14 anos, no contexto do EF de oito anos.

Entretanto, houve diferenças de sexo nas variáveis relacionadas ao desempenho acadêmico, às habilidades sociais e à medida de comportamento externalizante. Confirmando resultados de pesquisas prévias com alunos do $\mathrm{EF}$, as meninas apresentaram maiores médias no desempenho acadêmico (Grimm et al., 2010; Matthews et al., 2010) e em todas as habilidades sociais avaliadas (Bandeira et al., 2006; Matthews et al., 2010). Já os meninos apresentaram mais problemas de comportamento externalizante, o que também condiz com evidência previamente estabelecida (Fanti \& Henrich, 2010; Robbers et al., 2010; Silver et al., 2010). Segundo a perspectiva do funcionamento adaptativo na infância (Gardinal-Pizato et al., 2014; Masten \& Coatsworth, 1998), estes resultados sinalizam maior dificuldade dos meninos na adaptação às tarefas do $1^{\circ}$ ano do EF. A questão é retomada mais adiante nesta discussão.

A hipótese formulada na introdução foi amplamente confirmada. Em ambos os sexos, a presença de estresse foi inversamente associada com desempenho acadêmico e habilidades sociais, ao passo que se associou diretamente com problemas de comportamento. A relação inversa entre competência e estresse encontrada em ambos os sexos se alinha com o modelo teórico apresentado na introdução e concorda com os achados da investigação realizada por Trivellato-Ferreira e Marturano (2008) com alunos da antiga $1^{\mathrm{a}}$ série, que indicaram uma relação inversa dos sintomas de estresse com desempenho acadêmico e ajustamento. Nesse sentido, os resultados da presente pesquisa reiteram que crianças mais competentes apresentam maior facilidade para se ajustarem às demandas adaptativas da transição e, consequentemente, percebem as tarefas 
adaptativas como menos estressantes, o que as torna menos expostas aos efeitos adversos do estresse (Marturano, 2008).

A interpretação de um papel mediador da percepção de estressores na relação entre dificuldades adaptativas e sintomas de estresse é indiretamente corroborada pelos resultados que indicaram associação entre percepção de estressores e sintomas de estresse. Em ambos os sexos, houve associação da presença de estresse com a percepção de estressores escolares nos dois fatores, tanto em relação ao papel de estudante quanto nas relações interpessoais. Estes resultados reiteram a associação já estabelecida entre exposição a estressores escolares e sintomas de estresse (Hesketh et al., 2010; Hjern et al., 2008; Trivellato-Ferreira \& Marturano, 2008).

A esta altura da discussão, recoloca-se a questão de uma possível dificuldade maior dos meninos na adaptação às tarefas do $1^{\circ}$ ano do EF. De acordo com a linha de interpretação desenvolvida até aqui, os meninos, se de fato menos adaptados às demandas do $1^{\circ}$ ano, estariam apresentando mais sintomas de estresse, o que não ocorreu. No entanto, mostrou-se mais pronunciada no grupo masculino a associação entre presença de estresse e indicadores de realização das tarefas adaptativas. Como se pode verificar nas Tabelas 2 e 3, entre os meninos todas as 11 variáveis investigadas diferenciaram significativamente os grupos com e sem estresse na direção esperada, ao passo que entre as meninas foram seis as variáveis associadas ao estresse na direção prevista. Parece, assim, que os processos envolvidos na transição do $1^{\circ}$ ano do EF diferem entre meninos e meninas, porém, sua descrição requer modelos complexos, que vão além de diferenças no nível dos sintomas de estresse relatados pelas crianças.

Embora as escolhas metodológicas desta pesquisa tenham atendido ao seu principal objetivo, suas limitações podem ter contribuído para a permanência de lacunas sobre a investigação da associação do estresse à variável sexo. A fim de supri-las, sugere-se para futuras pesquisas a adoção de desenho prospectivo, com teste de modelos de predição do estresse compatíveis com a complexidade vislumbrada neste estudo.
Também pode ser útil o uso de outros métodos de avaliação de estresse, como, por exemplo, o diagnóstico clínico de estresse ou a identificação das fases de sua evolução, separadamente, conforme propostos por Lipp e Lucarelli (2008).

Outra limitação deste estudo refere-se à amostra, composta apenas por alunos de escolas municipais. A fim de aumentar o poder de generalização dos resultados, recomenda-se que estudos posteriores insiram na amostra alunos de outros tipos de escolas, como as estaduais e as particulares.

Por fim, dada a elevada taxa de sintomas de estresse na amostra, os achados sinalizam uma demanda por trabalhos de intervenção voltados à prevenção de problemas associados ao estresse em escolares. Ao identificar condições associadas à presença de estresse, eles também oferecem subsídios para tais práticas, com ênfase na promoção de habilidades sociais. Também parece fundamental o desenvolvimento de estratégias de coping para a criança lidar com as tensões escolares cotidianas, uma importante fonte de estresse, com repercussão na saúde emocional dos alunos no EF (Eriksson \& Sellströn, 2010), particularmente entre os mais jovens, como fator de risco para depressão e ansiedade (Byrne et al., 2011). Entretanto, investigações são necessárias para esclarecer os processos implicados nessas associações, testando modelos de predição com poder para detectar diferentes direções de efeitos entre as variáveis estudadas.

\section{Referências}

Achenbach, T. M., \& Edelbrock, C. S. (1979). The child behavior profile: II. Boys aged 12-16 and girls aged 6-11 and 12-16. Journal of Consulting and Clinical Psychology, 47(2), 223-233. doi:10.1037/0022-006X.47.2.223

Bandeira, M., Del Prette, Z. A. P., Del Prette, A., \& Magalhães, T. (2009). Validação das escalas de habilidades sociais, comportamentos problemáticos e competência acadêmica (SSRR-BR) para o ensino fundamental. Psicologia: Teoria e Pesquisa, 25(2), 271-282. doi:10.1590/S010237722009000200016

Bandeira, M., Rocha, S. S., Freitas, L. C., Del Prette, Z. A. P., \& Del Prette, A. (2006). Habilidades 
sociais e variáveis sociodemográficas em estudantes do ensino fundamental. Psicologia em Estudo, 11(3), 541-549. doi:10.1590/S141373722006000300010

Bridley, A., \& Jordan, S. S. (2012). Child routines moderate daily hassles and children's psychological adjustment. Children's Health Care, 41(2), 129-144. doi:10.1080/02739615.2012.65 7040

Byrne, D. G., Thomas, K. A., Burchell, J. L., Olive, L. S., \& Mirabito, N. S. (2011). Stressor experience in primary school-age children: Development of a scale to assess profiles of exposure and effects on psychological well-being. International Journal of Stress Management, 18(1), 88-111. doi:10.1037/a0021577

Chen, X., Huang, X., Chang, L., Wang, L., \& Li, D. (2010). Aggression, social competence, and academic achievement in Chinese children: A 5-year longitudinal study. Development and Psychopathology, 22(3), 583-592. doi:10.1017/ S0954579410000295

Correia-Zanini, M. R. G. (2013). Um estudo prospectivo sobre o percurso escolar de crianças nos primeiros anos do ensino fundamental (Tese de doutorado). Recuperado em http://www. teses.usp.br/teses/disponiveis/59/59137/tde06012014-100239/

Del Prette, Z. A. P., \& Del Prette, A. (2005). Psicologia das habilidades sociais na infância: Teoria e prática. Petrópolis, RJ: Vozes.

Enumo, S. R. F., Ferrão, E. S., \& Ribeiro, M. P. L. (2006). Crianças com dificuldade de aprendizagem e a escola: Emoções e saúde em foco. Estudos de Psicologia (Campinas), 23(2), 139-149. doi:10.1590/S0103-166X2006000200004

Eriksson, U., \& Sellström, E. (2010). School demands and subjective health complaints among Swedish schoolchildren: A multilevel study. Scandinavian Journal of Public Health, 38(4), 344-350. doi:10.1177/1403494810364683

Escobar, M., Alarcón, R., Blanca, M. J., Fernández-Baena, F. J., Rosel, J. F., \& Trianes, M. V. (2013). Daily stressors in school-age children: A multilevel approach. School Psychology Quartely, 28(3), 227-238. doi:10.1037/spq0000020

Fanti, K. A., \& Henrich, C. C. (2010). Trajectories of pure and co-occurring internalizing and externalizing problems from age 2 to age 12 :
Findings from the National Institute of Child Health and Human Development Study of Early Child Care. Developmental Psychology, 46(5), 1159-1175. doi:10.1037/a0020659

Gardinal-Pizato, E. C. (2010). Um estudo longitudinal de trajetórias de desempenho escolar (Tese de doutorado não publicada, Universidade de São Paulo, Ribeirão Preto, SP, Brasil).

Gardinal-Pizato, E. C., Marturano, E. M., \& Fontaine, A. M. G. V. (2014). Trajetórias de habilidades sociais e problemas de comportamento no ensino fundamental: Influência da educação infantil. Psicologia: Reflexão e Crítica, 27(1), 189-197. doi:10.1590/S0102-79722014000100021

Grimm, K. J., Steele, J. S., Mashburn, A. J., Burchinal, M., \& Pianta, R. C. (2010). Early behavioral associations of achievement trajectories. Developmental Psychology, 46(5), 976-983. doi: $10.1037 / \mathrm{a} 0018878$

Hesketh, T., Zhen, Y., Lu, L., Dong, Z.X., Jun, Y.X.,\& Xing, Z.W. (2010). Stress and psychosomatic symptoms in Chinese school children: Cross-sectional survey. Archives of Disease in Childhood, 95(2), 136-140. doi:10.1136/ adc. 2009.171660

Hjern, A., Alfven, G., \& Östberg, V. (2008). School stressors, psychological complaints and psychosomatic pain. Acta Paediatrica, 97(1), 112-117. doi:10.1111/j.1651-227.2007.00585.x

Instituto Nacional de Estudos e Pesquisas Educacionais Anísio Teixeira. (2009). Provinha Brasil: Kit Teste 2009. Brasília, DF: Autor. Recuperado em http://provinhabrasil.inep.gov.br

Kanner, A. D., Coyne, J. C., Schaefer, C., \& Lazarus, R. S. (1981). Comparison of two modes of stress measurement: Daily hassles and uplifts versus major life events. Journal of Behavioral Medicine, 4(1), 1-39. doi:10.1007/BF00844845

Lipp, M. E. N. (2000). O stress da criança e suas consequências. In M. E. N. Lipp (Ed.), Crianças estressadas: Causas, sintomas e soluções (pp. 13-42). Campinas, SP: Papirus.

Lipp, M. E. N., \& Lucarelli, M. D. M. (2008). Escala de Estresse Infantil (ESI): Manual. São Paulo, SP: Casa do Psicólogo.

Lipp, M. E. N., Arantes, J. P., Buriti, M. S., \& Witzig, T. (2002). O estresse em escolares. Psicologia Escolar e Educacional, 6(1), 51-56. doi:10.1590/ S1413-85572002000100006 
Marôco, J. (2011). Análise estatística com o SPSS Statistics (5. ed.). Pero Pinheiro, Portugal: Report Number.

Marturano, E. M. (2008). Tensões cotidianas na transição da primeira série: Um enfoque de desenvolvimento. Psicologia em Estudo, 13(1), 77-85. doi:10.1590/S1413-73722008000100010

Marturano, E. M., \& Gardinal, E. C. (2008). Um estudo prospectivo sobre o estresse cotidiano na $1^{\mathrm{a}}$ série. Aletheia, 27(1), 81-97. Recuperado em http:// pepsic.bvsalud.org/scielo.php?pid=S141303942008000100007\&script=sci_arttext

Masten, A. S., \& Coatsworth, J. D. (1998). The development of competence in favorable and unfavorable environments: Lessons from research on successful children. American Psychologist, 53(2), 205-220. doi:10.1037/0003066X.53.2.205

Matthews, J. S., Kizzie, K. T., Rowley, S. J., \& Cortina, K. (2010). African Americans and Boys: Understanding the literacy gap, tracing academic trajectories, and evaluating the role of learning-related skills. Journal of Educational Psychology, 102(3), 757-771. doi:10.1037/ a0019616

Pacanaro, S. V., \& Di Nucci, E. P. (2005). Stress infantil: Uma comparação entre meninos e meninas do ensino fundamental. Argumento, 7(13), 65-76. Recuperado em http://www.portal. anchieta.br/revistas-e-livros/argumento/pdf/ argumento13.pdf

Pereira, M. T., Marturano, E. M., Gardinal-Pizato, E. C., \& Fontaine, A. M. G. (2011). Possíveis contribuições da educação infantil no desempenho e competência social de escolares. Psicologia Escolar e Educacional, 15(1), 101-109. doi:10.1590/S1413-85572011000100011

Rende, R. D., \& Plomin, R. (1992). Relations between first grade stress, temperament, and behavior problems. Journal of Applied Developmental Psychology, 13(4), 435-446. doi:10.1016/01933973(92)90011-6
Robbers, S. C. C., Bartels, M., van Oort, F. V. A., van Beijsterveldt, C. E. M., van der Ende, J., Verhulst, F. C., ...Huizink, A. C. (2010). A twin-singleton comparison of developmental trajectories of externalizing and internalizing problems in 6- to 12-year-old children. Twin Research and Human Genetics, 13(1), 79-87. doi:10.1375/twin.13.1.79

Silver, R. B., Measelle, J. R., Armstrong, J. M., \& Essex, M. J. (2010). The impact of parents, child care providers, teachers, and peers on early externalizing trajectories. Journal of School Psychology, 48(6), 555-583. doi:10.1016/j. jsp.2010.08.003

Tanganelli, M. S. L., \& Lipp, M. E. N. (1998). Sintomas de stress na rede pública de ensino. Estudos de Psicologia (Campinas), 15(3), 17-27.

Trivellato-Ferreira, M. C., \& Marturano, E. M. M. (2008). Recursos da criança, da família e da escola predizem competência na transição da $1^{\mathrm{a}}$ série. Revista Interamericana de Psicologia, 42(3), 549-558. Recuperado em http://pepsic. bvsalud.org/pdf/rip/v42n3/v42n3a15.pdf

Vanaelst, B., De Vriendt, T., Huybrechts, I., Rinaldi, S., \& De Henauw, S. (2012). Epidemiological approaches to measure childhood stress. Paediatric and Perinatal Epidemiology, 26(3), 280297. doi:10.1111/j.1365-3016.2012.01258.x

Vilela, M.V. (1995). Sintomas e fontes de stress em uma amostra de escolares da $1^{a}$ a $4^{a}$ série (Dissertação de mestrado não publicada, Pontifícia Universidade Católica de Campinas, Campinas, SP, Brasil).
Recebido: 17/09/2015

$1^{a}$ revisão: $16 / 03 / 2016$

Aceite final: 18/03/2016 